\title{
Cyclin DI affects epithelial-mesenchymal transition in epithelial ovarian cancer stem cell-like cells
}

This article was published in the following Dove Press journal:

OncoTargets and Therapy

19 June 2013

Number of times this article has been viewed

\author{
Jie Jiao ${ }^{1,4}$ \\ Lu Huang' \\ Feng $\mathrm{Ye}^{\prime}$ \\ MinFeng Shi \\ XiaoDong Cheng ${ }^{3}$ \\ XinYu Wang ${ }^{3}$ \\ DongXiao $\mathrm{Hu}^{3}$ \\ Xing $\mathrm{Xie}^{3}$ \\ WeiGuo Lu $^{3}$ \\ 'Women's Reproductive Health \\ Laboratory of Zhejiang Province, \\ Women's Hospital, School of \\ Medicine, Zhejiang University, \\ Hangzhou, ${ }^{2}$ Department of \\ Gynaecology and Obstetrics, Changhai \\ Hospital, the Second Military Medical \\ University, Shanghai, ${ }^{\text {Women's }}$ \\ Reproductive Health Laboratory \\ of Zhejiang Province, Department \\ of Gynecologic Oncology, Women's \\ Hospital, School of Medicine, Zhejiang \\ University, Hangzhou, ${ }^{4}$ Department \\ of Gynaecology and Obstetrics, \\ Hangzhou First People's Hospital, \\ Hangzhou, People's Republic of China
}

Correspondence: WeiGuo Lu Women's Reproductive Health Laboratory of Zhejiang Province, Department of Gynecologic Oncology, Women's Hospital, School of Medicine, Zhejiang University, Xueshi Rd\#2,

Hangzhou, 310006 ,

People's Republic of China

Tel +8657 I 8703 II 30

Fax +8657187061878

Email Iwg@hzcnc.com
Background: The association of cancer stem cells with epithelial-mesenchymal transition (EMT) is receiving attention. We found in our previous study that EMT existed from CD24phenotype cells to their differentiated cells. It was shown that cyclin D1 functioned in sustaining self-renewal independent of CDK4/CDK6 activation, but its effect on the EMT mechanism in ovarian cancer stem cells is unclear.

Methods: The anchorage-independent spheroids from ovarian adenocarcinoma cell line $3 \mathrm{AO}$ were formed in a serum-free medium. CD24- and CD24+ cells were isolated by fluorescenceactivated cell sorting. Cell morphology, viability, apoptosis, and migratory ability were observed. Stem-related molecule Bmi-1, Oct-4 and EMT-related marker E-cadherin, and vimentin expressions were analyzed. Cyclin D1 expression in CD24- phenotype enriched spheroids was knocked down with small interfering RNA, and its effects on cell proliferation, apoptosis, migration ability, and EMT-related phenotype after transfection were observed.

Results: In our study, CD24- cells presented stronger proliferative, anti-apoptosis capacity, and migratory ability, than CD24+ cells or parental cells. CD24- cells grew with a scattered spindle-shape within 3 days of culture and transformed into a cobblestone-like shape, identical to CD24+ cells or parental cells at 7 days of culture. CD24- cells or spheroids highly expressed cyclin D1, Bmi-1, and vimentin, and seldom expressed E-cadherin, while CD24+ or parental cells showed the opposite expression. Furthermore, cyclin D1-targeted small interfering RNA resulted in decreased vimentin expression in spheroids. Transfected cells also exhibited an obvious decrease in cell viability and migration, but an increase in cell apoptosis.

Conclusion: Cancer stem cell-like cells possess mesenchymal characteristics and EMT ability, and cyclin D1 involves in EMT mechanism, suggesting that EMT of cancer stem cell-like cells may play a key role in invasion and metastasis of ovarian cancer.

Keywords: epithelial-mesenchymal transition, cancer stem cell, cyclin D1, ovarian cancer

\section{Introduction}

Epithelial ovarian carcinoma, accounting for approximately $90 \%$ of ovarian cancers, has always been the most lethal malignancy in the gynecological cancers. ${ }^{1}$ In order to explore the exact biological features of epithelial ovarian carcinoma, a series of studies is focusing on cancer stem cells or cancer stem cell-like cells (CSC-LCs). Cancer stem cells or CSC-LCs have been identified in several solid tumors in breast, liver, prostate, and other sites. ${ }^{2-5}$ However, in light of recent studies, ovarian cancer stem cells have been found to present diverse phenotypes and functions. ${ }^{6-9}$ Our previous study has identified that the CSC-LCs derived from the epithelial ovarian cancer cell line 3AO have CD24- phenotype with higher capacity of tumorigenesis, drug-resistance, selfrenewal, and differentiation abilities. ${ }^{10}$ 
The epithelial-mesenchymal transition (EMT) is the wellknown process whereby terminal differentiated epithelial cells convert into migratory mesenchymal cells in embryo development. ${ }^{11}$ Recently, EMT phenomenon in cancer stem cells is also been paid increasing attention. ${ }^{12}$ It was found that breast cancer stem cells with $\mathrm{CD} 44^{\text {high }} / \mathrm{CD} 24^{\text {low }}$ phenotype existed in the circulation system of breast cancer patients, and further, they expressed epithelial-mesenchymal transition markers, ${ }^{13}$ therefore this subset of cells was presumed to be the original source of breast cancer metastasis through EMT. Moreover, Mani et al ${ }^{14}$ discovered that a small group of cells with stem cell features could express CD $44^{\text {high }} / \mathrm{CD} 24^{\text {low }}$ phenotype by exogenous-induced EMT in the normal human and mouse mammary glands. It has been speculated that EMT may induce cancer cells to express stem cell phenotypes and thus acquire the abilities of migration and invasion. ${ }^{15}$ In addition, drug-resistance of cancer cells was also regarded to be associated with EMT. ${ }^{15}$ However, EMT in ovarian cancer stem cells and its effects on drug-resistance are still undiscovered. Preliminary findings in our previous study showed changed expression patterns of CK18 and Ep-CAM in $3 \mathrm{AO}$ spheroids and CD24- cells compared with parental cells and CD24+ cells respectively, implying the possibility that EMT existed from ovarian CSC-LCs to their differentiated cells. ${ }^{10}$

Proto-oncogenic cyclin D1 helps promote cell cycle progression by regulating the G1 to $\mathrm{S}$ phase. ${ }^{16} \mathrm{~A}$ recent study has showed that Cyclin D1 was necessary in maintenance of self-renewal in mammary stem and progenitor cells. ${ }^{17}$ Cyclin D1 functioned dependently on kinase 4 or 6 (CDK4 or CDK6) and ultimately induced phosphorylation and inactivation of tumor suppressor protein $\mathrm{Rb} .{ }^{18}$ It has been revealed that cyclin D1 also existed in different human tumors. ${ }^{19}$ Molenaar et al reported that neuroblastoma functionally depended on the overexpression of G1-regulating genes such as cyclin D1 to maintain its undifferentiated phenotype. ${ }^{20}$ It has also been shown that cyclin D1 overexpression may be correlated with platinum-resistance in ovarian, pancreatic, and non-small-cell lung carcinoma. ${ }^{16,21}$ A recent study found that cyclin D1 promoted survival of anchorage-independent cells beyond CDK4 or CDK6 pathways, ${ }^{22}$ which may play a critical role in tumorigenesis and cancer metastasis. However, to our knowledge, the effect of cyclin D1 on the EMT mechanism in cancer stem cells remains undiscovered. Here, we investigated the EMT phenomenon and cyclin D1 effect in ovarian CSC-LCs, and aimed to search and discover new targets for ovarian cancer therapy.

\section{Materials and methods Cell culture}

Ovarian adenocarcinoma cell line $3 \mathrm{AO}$ was obtained from the Women's Hospital, School of Medicine, Zhejiang University. Cells were maintained in RPMI 1640 medium, supplemented with $10 \%$ fetal bovine serum. The anchorage-independent spheroids were formed in a serum-free medium composed of DMEM/F12, $10 \mathrm{ng} / \mathrm{mL}$ basic fibroblast growth factor and $20 \mathrm{ng} / \mathrm{mL}$ epidermal growth factor (PeproTech Inc, Rocky Hill, NJ, USA), $1 \mathrm{mg} / \mathrm{mL}$ insulin (Sigma-Aldrich, St, Louis, $\mathrm{MO}, \mathrm{USA}$ ), and $10 \mu \mathrm{L} / \mathrm{mL} \mathrm{B} 27$ additive (Life Technologies, Carlsbad, CA, USA) on culture dishes. All cells were maintained at $37^{\circ} \mathrm{C}$ in a humidified $5 \% \mathrm{CO}_{2}$ incubator.

\section{Fluorescence-activated cell sorting analysis}

For fluorescence-activated cell sorting (FACS), 3AO adherent cells were washed twice with phosphate-buffered saline after $0.25 \%$ trypsin digestion. Cells were then suspended in phosphate-buffered saline and labeled with phycoerythrinconjugated mouse anti-human monoclonal CD24 antibody (Life Technologies). This was followed by FACS using a FACSAria flow cytometer (Beckman Coulter Inc, Indianapolis, IN, USA). Isotype control was established, and cells were routinely analyzed for purity.

\section{Cell viability assay}

Fresh CD24+ and CD24- cells were plated at 5000 per well onto 96-well plates with a low-serum medium (DMEM/ F12 supplemented with $1 \%$ fetal bovine serum) directly post-isolation and cultured overnight for cell attachment. At daily intervals (24, 48, 72, and 96 hours), $20 \mu \mathrm{L}$ MTT $(5 \mathrm{mg} / \mathrm{mL})$ were added per well and incubated in the dark for 4 hours. After removal of the medium, the dye crystals were dissolved in dimethyl sulfoxide for termination, and the absorbance was measured at OD490 with a Universal Microplate Reader $\mathrm{El}_{\mathrm{X}} 800$ (BioTek Instruments Inc, Winooski, VT, USA). Three independent experiments were done in quadruplicate wells.

\section{Cell apoptosis assay}

The parental adherent cells were treated with $50 \mu \mathrm{g} / \mathrm{mL}$ carboplatin (Bristol-Myers Squibb Co, New York, NY, USA) for 24 hours. Then, cells were digested with $0.25 \%$ trypsin without EDTA and washed twice with phosphatebuffered saline. Cells were colabeled with FITC-Annexin V (Biouniquer Technology, Beijing, People's Republic of China) and phycoerythrin-conjugated monoclonal CD24 antibody for 30 minutes at $4^{\circ} \mathrm{C}$ in the dark. Cell apoptosis of different 
cell subsets was analyzed using FACSAria flow cytometer. Propidium iodide was added 10 minutes before detection.

\section{Wound-repair assay}

Spheroids were trypsinized, then seeded $1 \times 10^{6}$ per well in 6-well plates overnight for adherent growth with four repeats. The same operation was applied to adherent parental cells. A wound was scratched in the middle of the monolayer with the tip of a sterile $10 \mu \mathrm{L}$ pipette, then a fresh serum-free medium was added. Ten representative fields of each group were marked and measured. Wounds were measured at 0,4 , and 8 hour intervals after scratching. The wound width was measured using a gauge tool with Photoshop CS4 software (Adobe Systems Inc, San Jose, CA, USA).

\section{RNA interference}

Small interfering RNA (siRNA) targeting cyclin D1 and nontargeting siRNA were both synthesized with the T7 RiboMAX ${ }^{\mathrm{TM}}$ Express RNAi System (Promega Corporation, Fitchburg, WI, USA). The cyclin D1-targeted mRNA sequence of cyclin D1 and the nontargeting control sequence were 5'-CCAGAGTGATCAAGTGTGA-3' and 5'-GACTTCATAAGGCGCATGC-3', respectively. ${ }^{23}$ DNA oligonucleotides used in siRNA synthesization are shown in Table 1. The process of siRNA synthesis was carried out strictly according to the technical bulletin. The interfering effect was routinely assessed at mRNA and protein level by quantitative reverse transcription polymerase chain reaction (qRT-PCR) and western blot. For siRNA transfection, the

Table I DNA oligonucleotides sequence used in synthesis of cyclin DI siRNA with T7 RiboMAX ${ }^{\mathrm{TM}}$ Express RNAi system

\begin{tabular}{ll}
\hline DNA oligonucleotides & $\mathbf{5}^{\prime}$-sequence-3' \\
\hline Oligo I (for cyclin DI siRNA) & GGATCCTAATACGACTCACTA \\
OACCAGAGTGATCAAGTGTGA
\end{tabular}

Abbreviations: DI siRNA, Cyclin DI small interfering RNA; NT siRNA, nontargeting small interfering RNA. spheroids were trypsinized and seeded $8 \times 10^{4}$ cells per well in 6-well plates with complete medium and were transfected with $80 \mathrm{nmol} / \mathrm{L}$ cyclin D1 siRNA or nontargeting siRNA using siPORT NeoFX Transfection Agent as a transfection mediator according to the manufacturer's instructions (Ambion, Carlsbad, CA, USA). To analyze the effect of cyclin D1 down-regulation on cell proliferation, cyclin D1 siRNAtransfected cells and negative control cells were plated 5000 per well in 96-well plates; cell viability was assessed by MTT assay as described above at 0, 24, 48, and 72 hour intervals after transfection. Cell apoptosis and wound-repair assays of transfected spheroid-differentiated cells within 3 day cultures were carried out as described above.

\section{Real-time RT-PCR analysis}

Total RNA was extracted using a Trizol reagent (Takara Bio Inc., Shiga, Japan) and reverse-transcribed into cDNA. The SYBR $^{\circledR}$ Premix ExTaq ${ }^{\mathrm{TM}}$ ( Takara Bio Inc.,) was used to carry out real-time RT-PCR with an Applied Biosystems 7900HT Fast real-time PCRsystem (Life Technologies) according to the manufacturer's protocols, and GAPDH was selected for normalization. Product specificity was confirmed by constructing a melting curve for each primer pair. All reactions were run in triplicate and no template control reactions were included in each assay run. A $\Delta c_{t}$ value means the differential value, which was derived by subtracting the ct value of each target gene from the $c_{t}$ value of GAPDH. The relative mRNA expression levels of all genes were calculated according to the $2^{-\Delta c t}$ method. The gene-specific primers for RT-qPCR are shown as Table 2 .

\section{Western blot analysis}

Cells were harvested into radioimmunoprecipitation assay lysate buffer containing freshly added 1\% phenylmethylsulfonyl fluoride. Protein was subjected to $10 \%$ SDSPAGE and then transferred to $0.45 \mu \mathrm{m}$ transfer membranes (Millipore, Billerica, MA, USA). Membranes were blocked at room temperature for 1 hour in Tris-buffered saline with Tween-20 containing 5\% nonfat milk, and then incubated with rabbit anti-cyclin D1 monoclonal antibody (1:5000), anti-N-cadherin $(1: 10,000)$ (Epitomics Inc, Burlingame, CA, USA), anti-vimentin (1:700; Abcam, Cambridge, UK), and mouse anti- $\beta$-actin monoclonal antibody (1:2000) (SigmaAldrich) at $4^{\circ} \mathrm{C}$ overnight. After incubation with appropriated secondary antibodies, goat-anti-mouse IgG (1:1000) or goatanti-rabbit IgG (1:1000) (Sigma-Aldrich), at room temperature for 1 hour, bands were visualized by Chemiluminescence Detection Kit for HRP (Biological Industries, Kibbutz Beit 
Table 2 Primer used for quantitative RT-PCR

\begin{tabular}{|c|c|c|}
\hline $\begin{array}{l}\text { Gene } \\
\text { name }\end{array}$ & Sequence $\left(5^{\prime}\right.$ to $\left.3^{\prime}\right)$ & Gene ID \\
\hline GAPDH & $\begin{array}{l}\text { Forward primer: } \\
\text { GACAGTCAGCCGCATCTTCT } \\
\text { Reverse primer: } \\
\text { TTAAAAGCAGCCCTGGTGAC }\end{array}$ & NM_002046.3 \\
\hline Oct-4 & $\begin{array}{l}\text { Forward primer: } \\
\text { GACAACAATGAAAATCTTCAGGAGA } \\
\text { Reverse primer: } \\
\text { TTCTGGCGCCGGTTACAGAACAA }\end{array}$ & NM_00II59542.I \\
\hline Bmi-I & $\begin{array}{l}\text { Forward primer: } \\
\text { ATGTGTGTGCTTTGTGGAG } \\
\text { Reverse primer: } \\
\text { AGTGGTCTGGTCTTGTGAAC }\end{array}$ & NM_005I80.6 \\
\hline E-cadherin & $\begin{array}{l}\text { Forward primer: } \\
\text { TGCCCAGAAAATGAAAAAGG } \\
\text { Reverse primer: } \\
\text { GTGTATGTGGCAATGCGTTC }\end{array}$ & NM_004360.3 \\
\hline Vimentin & $\begin{array}{l}\text { Forward primer: } \\
\text { GAGAACTTTGCCGTTGAAGC } \\
\text { Reverse primer: } \\
\text { GCTTCCTGTAGGTGGCAATC }\end{array}$ & NM_003380.3 \\
\hline Cyclin DI & $\begin{array}{l}\text { Forward primer: } \\
\text { TTCGGGATGATTGGAATAGC } \\
\text { Reverse primer: } \\
\text { TGTGAGCTGGCTTCATTGAG }\end{array}$ & NM_053056.2 \\
\hline
\end{tabular}

Abbreviations: RT-PCR, reverse transcription polymerase chain reaction; GAPDH, Glyceraldehyde 3-phosphate dehydrogenase.

Haemek, Israel). The densitometry readings of the bands were normalized to $\beta$-actin expression.

\section{Statistical analysis}

Homogeneity of variance and normal distribution were commonly tested. Appropriate statistical methods were used as needed. Student's $t$-test was used for measurement data with normal distribution, while the Mann-Whitney nonparametric test was used for data with nonnormal distribution. The level of significance was set at $P<0.05$.

\section{Results \\ CD24- cells possess stronger proliferative capacity}

The parental 3AO cells, CD24- and CD24+ cells with high purity underwent normal proliferation when seeded in the medium supplied with $1 \%$ fetal bovine serum within 48 hours; however, the proliferation rate of CD24+ cells was obviously lower than that of parental $3 \mathrm{AO}$ and CD24- cells. At 48 hours after culture, CD24+ cells stopped proliferating, while the other two kinds of cells still continuously proliferated. But at 72 hours, CD24- cells grew continuously while parental cells grew slowly and entered growth plateau (Figure 1A).

\section{CD24- cells present stronger resistance to platinum}

CD24- and CD24+ cells presented the obvious differences in resistance to carboplatin-induced apoptosis after 24 hours of carboplatin treatment (Figure 1B). The basic apoptosis level was not different between CD24- and CD24+ cells, while the nonviable apoptotic rate of CD24+ cells was significantly increased at 24 hours after carboplatin treatment compared with that of CD24- cells $(Z=-3.363$, $P=0.001)$ though the viable apoptotic rate was not found to be changed at $24 \mathrm{~h}(\mathrm{Z}=-0.211, P=0.878)$, as shown in Figure $1 \mathrm{~B}$ and $\mathrm{C}$.

\section{CD24- cells highly expressed stem- related gene Bmi-I}

Bmi-1 mRNA expression was significantly higher in fresh CD24- cells than that in fresh CD24+ cells $(t=4.761$, $P=0.001)$ and gradually and significantly decreased during the differentiation cultivation $(\mathrm{F}=11.584, P=0.001)$. However no similar change of Bmi-1 mRNA expression in CD24+ cells was found during the differentiation cultivation ( $\mathrm{F}=0.242, P=0.788)$. Oct-4 expression was not different between fresh CD24- and CD24+ cells ( $t=0.296$, $P=0.774)$, but gradually and significantly decreased in both cells during the differentiation cultivation $\left(\mathrm{F}_{\mathrm{CD} 24-}=6.016\right.$, $\left.P_{\mathrm{CD} 24-}=0.016 ; \mathrm{F}_{\mathrm{CD} 24+}=5.426, P_{\mathrm{CD} 24+}=0.021\right)$, as shown in Figure 1D.

\section{CD24- cells possessed EMT characteristic}

CD24- and CD24+ cells with high purity showed different growth features after isolation. CD24+ cells presented the typical cobblestone-like shape similar to parental cells, which grew as a single cell colony. But a confluence of monoclone gradually occurred following prolonged culture. Contrarily, CD24- cells grew with scattered spindle shape similar to fibroblast cells until 3 days of culture, but the cell colony grew towards the shape of parental $3 \mathrm{AO}$ cells following culture prolongation (Figure 2A).

The qRT-PCR assay revealed that E-cadherin mRNA expression in CD24- cells was significantly lower than CD24+ cells $(t=-4.095, P=0.015)$. There was a clear, upregulated trend of E-cadherin mRNA expression from fresh isolated, 3 -day cultures, to 7-day cultured CD24- cells ( $\mathrm{F}=6.459$, $P=0.012$ ), but not among CD24+ cells with different culture times. Vimentin mRNA expression in CD24- cells was significantly higher than CD24+ cells $(t=5.767, P=0.002)$. There was also a clear downregulated trend of vimentin 

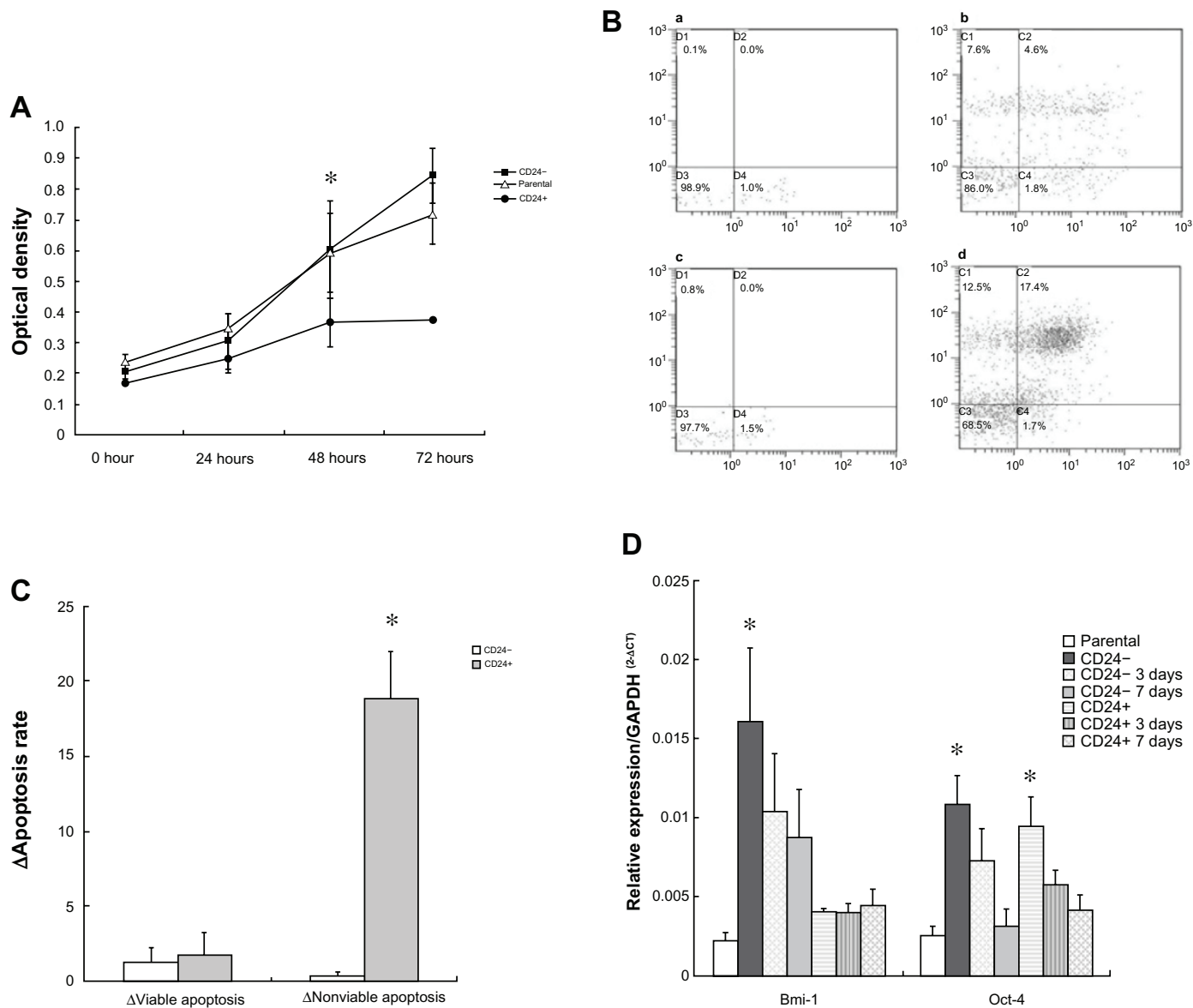

Figure I Cell viability, apoptosis, and stem-related genes expression in CD24- and CD24+ cells.

Notes: (A) The proliferative rate of CD24+ cells was obviously lower than that of parental 3AO and CD24- cells. At 48 hours after culture, CD24+ cells showed lower proliferation than the other two kinds of cells. At 72 hours after culture, only CD24- cells still persisted in proliferation. (B: a) CD24- cells before dosing, (B: b) CD24cells after dosing, (B: c) CD24+ cells before dosing, (B: d) CD24- cells after dosing. (C) Comparison of viable and nonviable apoptosis rates between CD24- and CD24+ cells. Nonviable apoptosis rate of CD24+ cells was significantly increased beyond that of CD24- cells $(Z=-3.363, P=0.00 \mathrm{I})$. (D) Bmi-I mRNA expression was significantly higher in CD24- cells than that in CD24+ cells $(t=4.76 \mathrm{I}, P=0.00 \mathrm{I})$, but gradually and significantly decreased during the differentiation cultivation $(\mathrm{F}=\mathrm{II} .584, P=0.00 \mathrm{I})$; Oct-4 expression was not significantly different between CD24- and CD24+ $(t=0.296, P=0.774)$, but gradually decreased during the differentiation cultivation in both cells $\left(\mathrm{F}_{\mathrm{CD} 24-}=6.016, P_{\mathrm{CD} 24-}=0.016 ; \mathrm{F}_{\mathrm{CD} 24+}=5.426, P_{\mathrm{CD} 24+}=0.021\right)$.

mRNA expression from fresh isolated, 3-day cultures, to 7-day cultured CD24- cells $(\mathrm{F}=54.637, P=0.001)$, but also not among CD24+ cells with different culture times (Figure 2B).

\section{CD24- enriched spheroids possess stronger migratory capability}

Our previous outcome has verified that an average of $80 \%$ of cells presented CD24- phenotype in spheroids cultured for 6 days. ${ }^{10}$ Wound assays were performed to compare the migratory potential between adherent parental cells and spheroid-differentiated cells. The width of wound closure was shown in Figure 2A. Spheroid-differentiated cells presented wider width closure than parental cells, and the difference was significant at 8 hours after inflicting a scratch $(Z=-4.099, P=0.000)$ though was not at
4 hours after scratching $(Z=-1.848, P=0.068)$ as shown in Figure $2 \mathrm{C}$ and $\mathrm{D}$.

\section{Spheroids highly express cyclin DI and vimentin}

The expression of cyclin D1, N-cadherin, and vimentin in parental and spheroids was detected by western blot. The average value of cyclin D1 was $0.507 \pm 0.069$ in parental cells and $1.007 \pm 0.132$ in spheroids, with a significant difference $(t=-3.351, P=0.007)$. The value of $\mathrm{N}$-cadherin was $0.501 \pm 0.063$ in parental cells and $0.599 \pm 0.039$ in spheroids without statistical difference $(t=-1.322, P=0.221)$. The average value of vimentin was $0.987 \pm 0.155$ in parental cells and $1.705 \pm 0.245$ in spheroids with a significant difference $(t=-2.471, P=0.033)$, as shown in Figure $3 \mathrm{~A}$ and $\mathrm{B}$. 
A
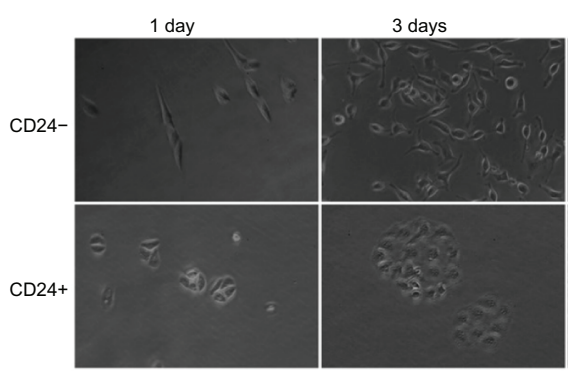

C
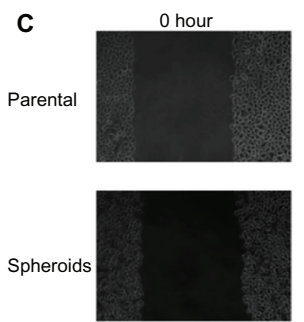
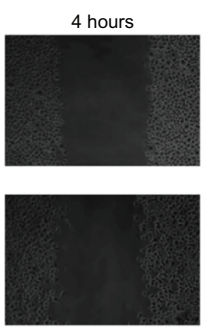

7 days
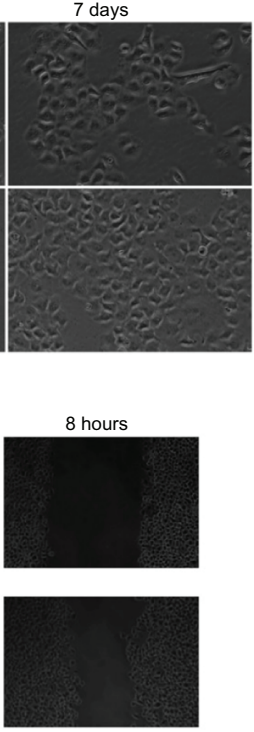

B
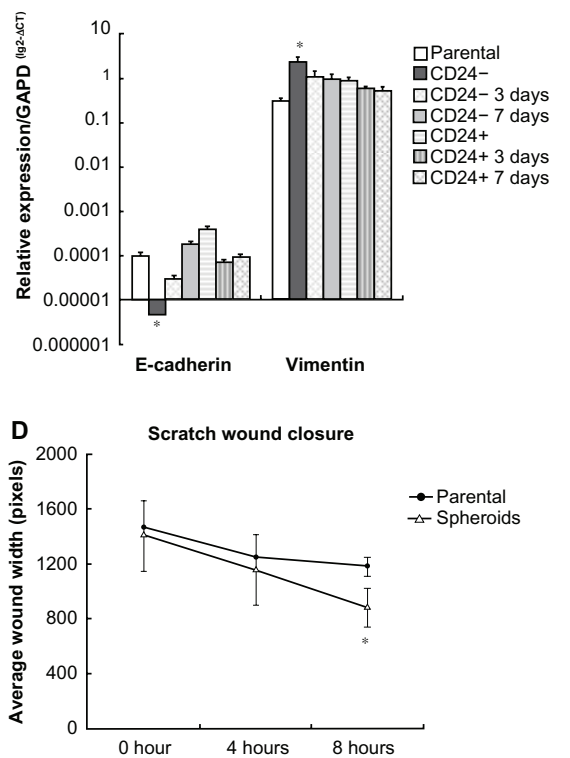

Figure 2 Epithelial-mesenchymal transition (EMT) phenomenon in CD24- cells.

Notes: (A) CD24- grew in the shape of scattered spindle, similar to fibroblast cells within 3 days of culture with complete medium, while CD24+ presented the typical cobblestone-like shape growth. Cell colonies grew towards the shape of parental $3 \mathrm{AO}$ cells as cultures were prolonged. (B) E-cadherin and vimentin expression in different cells. E-cadherin mRNA expression in CD24- cells was significantly lower than CD24+ cells $(t=-4.095, P=0.015)$. There was a clear upregulated trend of E-cadherin mRNA expression from fresh isolated, 3-day culture, to 7-day culture CD24- cells ( $F=6.459, P=0.012)$, but not among CD24+ cells with different culture times. Vimentin mRNA expression in CD24- cells was significantly higher than CD24+ cells $(t=5.767, P=0.002)$. There was also a clear downregulated trend of vimentin mRNA expression from fresh isolated, 3-day cultures, to 7-day culture CD24- cells $(F=54.637, P=0.00 \mathrm{I})$, but not among CD24+ cells with different culture times. (C and $\mathbf{D})$ The wound-repair assay: spheroid-differentiated cells revealed significantly increased width closure than parental adherent cells at 4 hours $(P=0.068)$ and at 8 hours after scratch $(P=0.000)$.

A

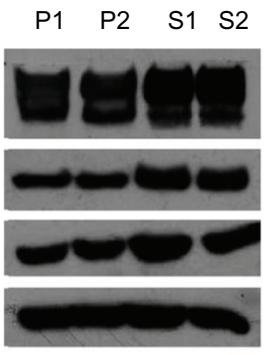

B

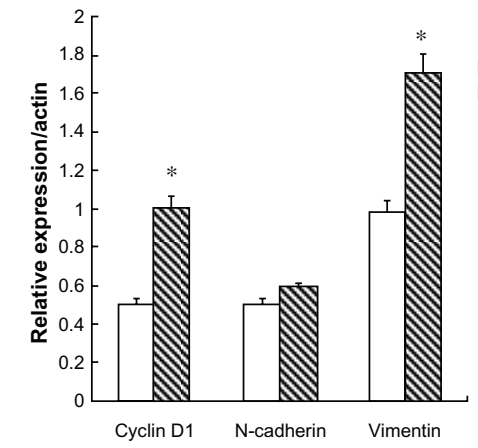

Cyclin D1 siRNA Scramble siRNA Control

D

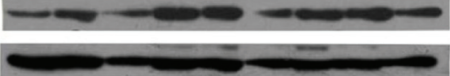

Cyclin D1

$\beta$-actin

C

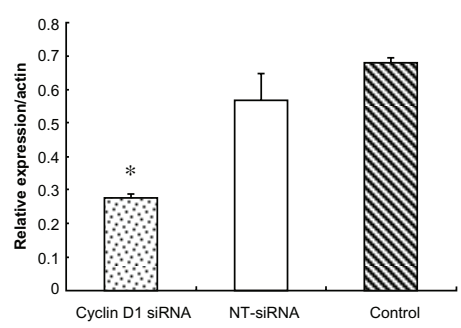

Figure 3 The expression pattern of cyclin DI, N-cadherin, vimentin in parental cells and spheroids, and transfection efficacy of cyclin DI siRNA.

Notes: $(\mathbf{A}$ and $\mathbf{B})$ The average value of cyclin DI was higher in spheroids than that in parental cells with a significant difference $(t=-3.35 \mathrm{I}, P=0.007)$. The value of $\mathrm{N}$-cadherin was not statistically different between spheroids and parental cells $(t=-1.322, P=0.22 \mathrm{I})$. The average value of vimentin was also higher in spheroids than that in parental cells, with a significant difference $(t=-2.47 \mathrm{I}, P=0.033)$. (C) Cyclin DI mRNA was downregulated nearly 59\% after siRNA transfection. (D) Cyclin DI protein was downregulated nearly $51 \%$ after siRNA transfection.

Abbreviations: PI, parental cell sample I; P2, parental cell sample 2; PI, spheroids sample I; S2, spheroids sample 2; NT-siRNA, nontargeting small interfering RNA. 


\section{Cyclin DI knockdown alters} the proliferation, apoptosis, and migration abilities of spheroid-differentiated cells

At 48 hours after cyclin D1 siRNA was transfected into spheroid-differentiated cells, the amount of cyclin D1 mRNA was decreased by $59.13 \%$ and cyclin D1 protein was reduced by $51.76 \%$ compared with the control group (Figure 3C and D).

Cell viability was observed after cyclin D1 knockdown. MTT assay showed that the cell viability was not obviously changed at 0,24 , and 48 hours, but significantly decreased at 72 hours after cyclin D1 siRNA transfection $(Z=-2.241$, $P=0.025)$, as shown in Figure 4A.

The effect of cyclin D1 knockdown on cell apoptosis was observed by FITC-Annexin V/Propidium iodide apoptosis assay. As shown in Figure 4B and C, at 30 hours after siRNA transfection, the viable apoptosis was significantly increased in the cyclin D1 siRNA-transfected cells, compared with scramble siRNA group $(Z=-2.650$, $P=0.008)$.

A

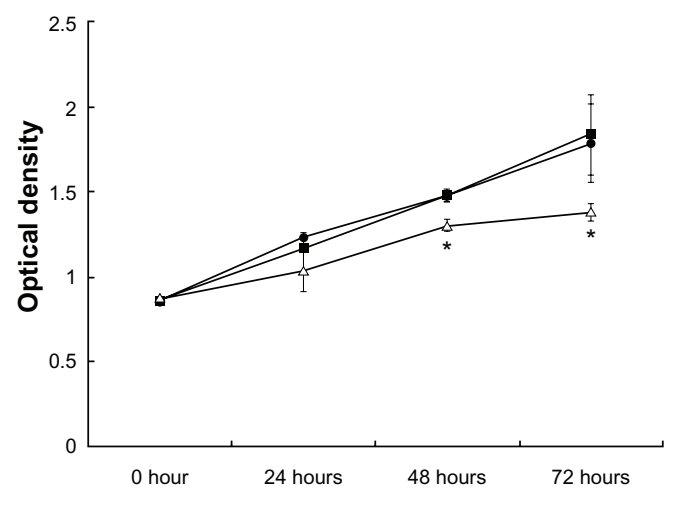

The change of migration ability of spheroid-differentiated cells after cyclin D1 knockdown was further observed. Spheroiddifferentiated cells were plated $8 \times 10^{4}$ per well in 6-well plates. Following attachment overnight, cells were transfected with cyclin D1 siRNA. At $12 \mathrm{~h}$ after transfection, cells were replated $3 \times 10^{5}$ per well in 12 -well plates for attachment overnight, then wound repair assays were performed. At 16 hours after inflicting a scratch, wound width of cyclin D1 siRNA-transfected cells was significantly decreased compare to that of control $(t=-3.125, P=0.004)$ as shown in Figure $5 \mathrm{~A}$ and $\mathrm{B}$.

\section{Cyclin DI knockdown reduces the expression of vimentin and $\mathrm{N}$-cadherin protein in spheroid-differentiated cells}

Knockdown of cyclin D1 resulted in significantly decreased expression of vimentin protein in spheroid-differentiated cells $(t=3.748, P=0.002)$. $\mathrm{N}$-cadherin expression was also decreased, but the difference was not significant $(\mathrm{Z}=-1.083$, $P=0.279$ ), as shown in Figure 5C and D.

B
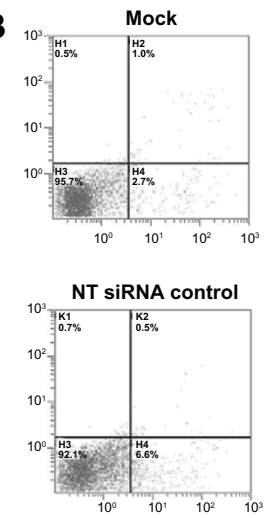
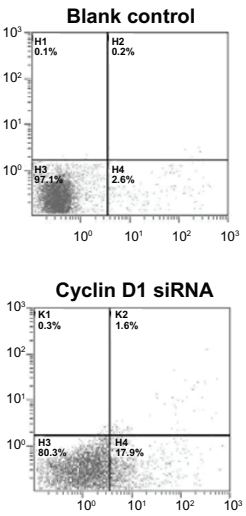

C

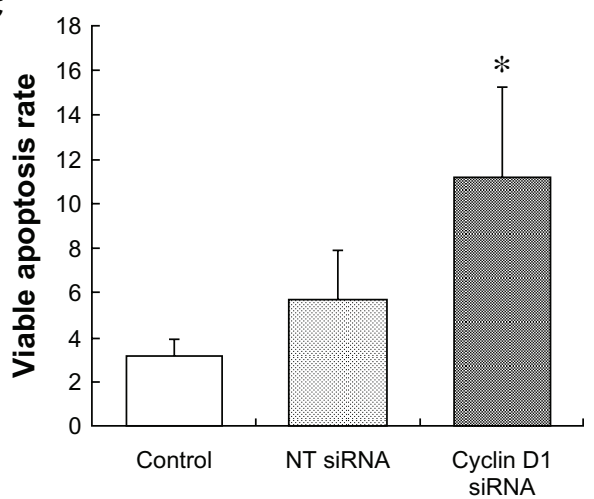

Figure 4 Cell viability, apoptosis induced by cyclin DI silence.

Notes: (A) At 0, 24, and 48 hours, cell viability was not significantly different between cyclin DI-siRNA and NT siRNA transfected cells, but a marked decrease of cell viability was observed at 72 hours post-transfection $(Z=-2.24 I, P=0.025)$. (B and $\mathbf{C})$ At 30 hours after siRNA transfection, viable apoptosis was significantly increased in cyclin $D I$ siRNA-transfected cells compared with NT siRNA group $(Z=-2.650, P=0.008)$.

Abbreviation: NT-siRNA, nontargeting small interfering RNA. 


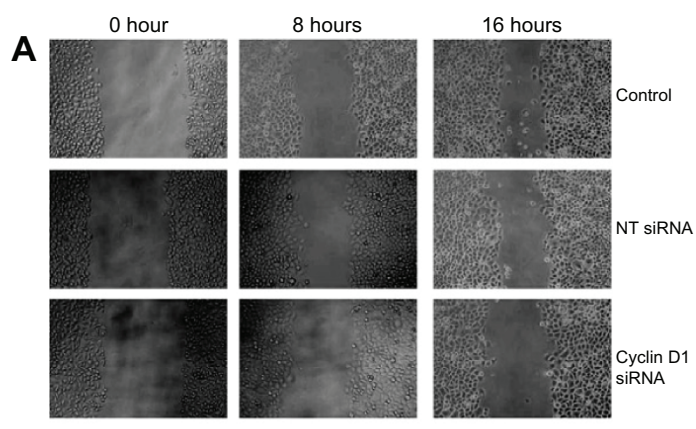

C

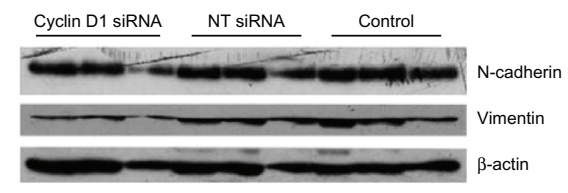

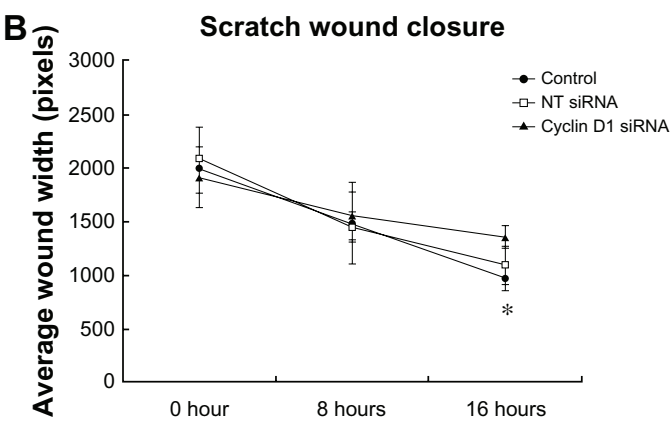

D

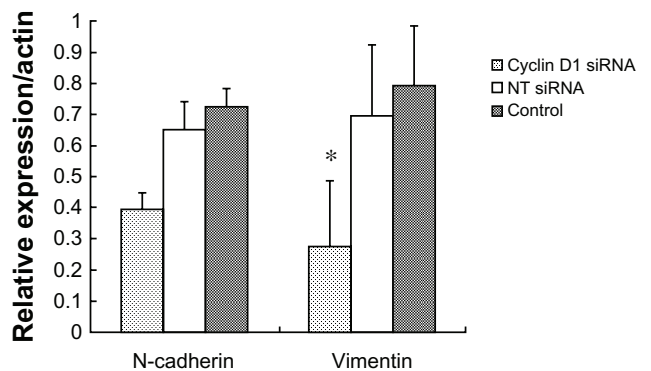

Figure 5 Cell migration and $\mathrm{N}$-cadherin, vimentin expression induced by cyclin DI silence.

Notes: (A and B) Wound width of cyclin DI siRNA-transfected cells was significantly decreased compared to that of control $(t=-3.125, P=0.004)$; $(\mathbf{C}$ and $\mathbf{D})$ Knockdown of cyclin DI resulted in significantly decreased expression of vimentin protein in spheroid-differentiated cells $(t=3.748, P=0.002)$. N-cadherin expression was not significantly different $(Z=-1.083, P=0.279)$.

Abbreviation: NT-siRNA, nontargeting small interfering RNA.

\section{Discussion}

It has been well recognized that cancer stem cells possess the following characteristics: (a) strong clone formation ability in vitro with self-renewal capability, (b) suspension spheroid formation in vitro, (c) expression of several known stem cellrelated markers, such as Oct-4, Nanog, and others, and (d) propagation in animals and recapitulation of their original phenotype. ${ }^{6,24,25}$ As shown in our study, CD24- cells derived from ovarian epithelial adenocarcinoma cell line $3 \mathrm{AO}$ were able to proliferate normally under low serum conditions, suggesting that CD24- cells possess much stronger proliferative ability than parental and CD24+ cells. Previous studies have revealed that cancer stem cells only account for a very small proportion of tumor tissue, and usually stay in a state of quiescence. When damage occurs within the extracellular environment, such as starvation conditions of low or free serum and toxicity conditions of chemotherapy drugs, those maturely differentiated cells hardly tolerate such environmental pressures, presenting growth arrest and apoptosis acceleration, even death. ${ }^{13,26,27}$ In contrast, the cancer stem cells can break through the quiescent state and into proliferation. Several studies have shown evidence that some phenotypes of cancer stem-like cells may be induced by long-term chemotherapy drugs stimulation. ${ }^{13,26,27}$ As in our study, CD24- cells always showed continuous growth under $1 \%$ low serum, while others presented slower growth despite sustaining proliferation for a short period. Besides, parental cells appeared to possess slightly stronger proliferation ability than CD24+ cells, which may be associated with the existence of a small proportion of cancer stem cells in the parental cells. Furthermore, we found that CD24- cells showed a more significant decrease in nonviable apoptosis rate than did CD24+ cells though their basic viable apoptosis and viable apoptosis rate after dosing were the same. Thus, our results further support the findings that cancer stem cells possess stronger survival ability against extraneous damage.

The expression of undifferentiated markers is one of the most important features of cancer stem cells. We analyzed the expression of stem cell-related markers in CD24- and CD24+ cells, such as Bmi-1 and Oct-4, which have been regarded to be associated with stem and/or progenitor cells and function essential for the maintenance of an undifferentiated state. ${ }^{28-33}$ Bmi-1 was highly expressed in CD24- cells but gradually downregulated following cell differentiation, suggesting that Bmi-1 functioned with specificity for sustaining selfrenewal characteristics in ovarian cancer stem cell-like cells. However, we found that Oct-4 was expressed in both CD24+ and CD24- cells, though it was downregulated following culture prolongation. We speculate therefore that Oct-4 may not be the specific marker for ovarian cancer stem cells, but further evidence is needed. 
Epithelial cells have long been regarded as cells of terminal differentiation, but recent studies have found that epithelial cells can lose adhesion, tight junction, and cell polarity to acquire the ability of invasive migration under the action of some factors. ${ }^{6,24,25}$ Meanwhile, these epithelial cells still present morphology and properties of mesenchyme cells. EMT is a necessary physical phenomenon of mammal embryonic development process. It has been verified that EMT is a main way by which embryonic stem cells obtain migrating ability. ${ }^{34}$ Accumulated evidence has also revealed that EMT is closely related with the occurrence and development of malignant epithelial tumors, ${ }^{35,36}$ and plays a pivotal role in primary invasion and secondary metastasis of cancers, including breast, prostate, hepatic cancer, melanoma, and others. ${ }^{13,37-39}$ In this study, we found that CD24- cells, freshly isolated with high purity, presented the shape of scattered spindle similar to fibroblast cells within 3 days of culture, while CD24+ and parental cells grew in the typical cobblestone-like shape. But morphology of CD24- cells changed into the shape similar to CD24+ and parental cells following cell differentiation, and part of them presented the typical cobblestone-like shape when culture continued to 7 days. Our results suggest that a differentiated culture of CD24- cells possesses the ability to transit morphologically from mesenchymal back to epithelial features.

During the EMT process, epithelial cells need to reduce or lose key proteins of epithelial cell adhesion junctions and tight junctions, and re-express or upregulate proteins originated from mesenchymal cells. E-cadherin and vimentin protein are considered two key proteins related to EMT. E-cadherin belongs to type I cadherin, which not only constitutes the powerful intercellular connection, but also maintains epithelial cell characteristics. The loss of E-cadherin is usually accompanied by the occurrence of nonepithelial cell features, including loss of cell polarity. Vimentin is one of the main components of secondary fibers in fibroblasts. Previous studies reported that the loss or downregulation of E-cadherin and upregulation of vimentin occurred almost simultaneously during the EMT process. ${ }^{13,37-39}$ In this study, we found that fresh CD24- cells presented high expression of vimentin mRNA and protein and downregulated mRNA level following culture maintenance. Contrarily, CD24- cells expressed very low E-cadherin mRNA. Although there was a clear upregulated trend of E-cadherin mRNA expression following cell differentiation, we still could not detect E-cadherin protein by western blot. Whether ovarian epithelial cells express E-cadherin remains controversial. Some investigators reported that the original cadherin in the normal ovarian surface epithelium was
$\mathrm{N}$-cadherin, but not E-cadherin; however, others showed that only some clones expressed E-cadherin. ${ }^{40-42}$ The causation is not clear and might be associated with the existence of modified or instable expression at the protein level. Nevertheless, our results suggest that $\mathrm{CD} 24$ - ovarian cancer stem-like cells possess mesenchymal phenotypes and EMT potential, at least at mRNA level.

Wound-repair assays can be used for measuring the migration ability of cells and have been recently shown to be useful for evaluating the mesenchymal feature. We adopted CD24phenotype enriched spheroid cells for study and found that spheroid-differentiated cells showed significantly stronger wound-repair ability than parental cells both at 4 hours and 8 hours after inflicting a scratch; although, those cells had partly differentiated in spheroids. Hence, we deduced that undifferentiated CD24- cells should possess extraordinarily stronger migratory ability. Our result is consistent with the mesenchymal feature of cancer stem cells.

Under normal circumstances, cell survival relies on adhesion to an extracellular matrix. Loss of the extracellular matrix support usually triggers a special apoptosis, termed anoikis. ${ }^{43}$ As shown in our study, cancer stem cells can survive as anchorage-independent spheroid forms under serum-free media conditions, which suggests that cancer stem cells have the capacity to acquire anoikis-resistance. It has been speculated that cyclin D1 may mediate this anoikis-resistance. Gan et $\mathrm{al}^{22}$ found that the oncoprotein, cyclin D1, acted as a critical upstream inhibitor of anoikis mediated by FOXO, one of the integrin family members, through an independent pathway rather than combination with CDK4 or CDK6. Furthermore, cyclin D1 is associated with platinum resistance, growth, and metastasis in various cancers including ovarian cancers. ${ }^{23}$ A recent study also found that cyclin D1 activity was required for the self-renewal of mammary stem and progenitor cells. ${ }^{17}$ Thus, we speculated that cyclin D1 probably played a role in EMT in cancer stem cells. In this study, we found that spheroids expressed higher cyclin D1 and vimentin than parental cells. Cyclin D1 gene silence could downregulate the expression of vimentin and N-cadherin. Moreover, siRNA targeting-cyclin D1 transfection could decelerate cell viability and migration ability and accelerate apoptosis compared with the negative control group. Our results suggest that cyclin D1 is involved in sustaining the mesenchymal feature of ovarian cancer stem cell-like cells in EMT.

\section{Conclusion}

Taken together, our results showed that CD24- cells and CD24- phenotype enriched spheroids from the ovarian cancer 
cell line 3AO presented mesenchymal features, such as overexpression of stem cell-related markers, higher proliferation, increased drug-resistance, lower apoptosis, and stronger migration capacity, and showed EMT phenomenon including morphology, phenotype, and function through differentiative culture. In addition, cyclin D1 is involved in EMT. Our findings suggest that ovarian CSC-LCs possess mesenchymal characteristics and EMT ability, which may play a key role in the invasion and metastasis of ovarian cancer.

\section{Acknowledgments}

This research was supported by the National Natural Science Foundation of China (Grant No 30973380), Zhejiang Provincial Natural Science Foundation of China (Grant No LY12H16024), Foundation of Health Department of Zhejiang Province of China (Grant No 2007A129), Specialized Research Fund for the Doctoral Program of Higher Education (Grant No 20070335060), National High-tech R\&D Program (863 Program) (Grant No 2012AA02A507), and by Zhejiang Provincial Program for the Cultivation of High-level Innovative Health Talents.

J Jiao, F Ye, WG Lu, and X Xie contributed to conception and design. J Jiao carried out the main parts of the study and the statistical analysis. WG Lu contributed to the revision of the article. MF Shi, L Huang, XY Wang, DX Hu and XD Cheng all participated in the data acquisition and analysis. All authors contributed to writing the manuscript and approved the final edition.

\section{Disclosure}

The authors report no conflicts of interest in this work.

\section{References}

1. Nossov V, Amneus M, Su F, et al. The early detection of ovarian cancer: from traditional methods to proteomics. Can we really do better than serum CA-125? Am J Obstet Gynecol. 2008;199(3):215-223.

2. Al-Hajj M, Wicha MS, Benito-Hernandez A, Morrison SJ, Clarke MF. Prospective identification of tumorigenic breast cancer cells. Proc Natl Acad Sci U S A. 2003;100(7):3983-3988.

3. Guzmán-Ramírez N, Völler M, Wetterwald A, et al. In vitro propagation and characterization of neoplastic stem/progenitor-like cells from human prostate cancer tissue. Prostate. 2009;69(15):1683-1693.

4. Zhu Z, Hao X, Yan M, et al. Cancer stem/progenitor cells are highly enriched in CD133+ CD44+ population in hepatocellular carcinoma. Int J Cancer. 2010;126(9):2067-2078.

5. Singh SK, Hawkins C, Clarke ID, et al. Identification of human brain tumour initiating cells. Nature. 2004;432(7015):396-401.

6. Zhang $\mathrm{S}$, Balch $\mathrm{C}$, Chan $\mathrm{MW}$, et al. Identification and characterization of ovarian cancer-initiating cells from primary human tumors. Cancer Res. 2008;68(11):4311-4320.

7. Kusumbe AP, Mali AM, Bapat SA. CD133-expressing stem cells associated with ovarian metastases establish an endothelial hierarchy and contribute to tumor vasculature. Stem Cells. 2009;27(3):498-508.
8. Hu C, Li H, Li J, et al. Analysis of ABCG2 expression and side population identifies intrinsic drug efflux in the HCC cell line MHCC-97L and its modulation by Akt signaling. Carcinogenesis. 2008;29(12): 2289-2297.

9. Szotek PP, Pieretti-Vanmarcke R, Masiakos PT, et al. Ovarian cancer side population defines cells with stem cell-like characteristics and mullerian inhibiting substance responsiveness. Proc Natl Acad Sci U S A. 2006;103(30):11154-11159.

10. Shi MF, Jiao J, Lu WG, et al. Identification of cancer stem cell-like cells from human epithelial ovarian carcinoma cell line. Cell Mol Life Sci. 2010;67(22):3915-3925.

11. Yang J, Weinberg RA. Epithelial-mesenchymal transition: at the crossroads of development and tumor metastasis. Dev Cell. 2008;14(6):818-829.

12. Singh A, Settleman J. EMT, cancer stem cells and drug resistance: an emerging axis of evil in the war on cancer. Oncogene. 2010;29(34): $4741-4751$.

13. Aktas B, Tewes M, Fehm T, Hauch S, Kimmig R, Kasimir-Bauer S. Stem cell and epithelial-mesenchymal transition markers are frequently overexpressed in circulating tumor cells of metastatic breast cancer patients. Breast Cancer Res. 2009;11(4):R46.

14. Mani SA, Guo W, Liao MJ, et al. The epithelial-mesenchymal transition generates cells with properties of stem cells. Cell. 2008;133(4): 704-715.

15. Polyak K, Weinberg RA. Transitions between epithelial and mesenchymal states: acquisition of malignant and stem cell traits. Nat Rev Cancer. 2009;9(4):265-273.

16. Biliran $\mathrm{H} \mathrm{Jr}$, Wang $\mathrm{Y}$, Banerjee $\mathrm{S}$, et al. Overexpression of cyclin D1 promotes tumor cell growth and confers resistance to cisplatin-mediated apoptosis in an elastase-myc transgene-expressing pancreatic tumor cell line. Clin Cancer Res. 2005;11(16):6075-6086.

17. Jeselsohn R, Brown NE, Arendt L, et al. Cyclin D1 kinase activity is required for the self-renewal of mammary stem and progenitor cells that are targets of MMTV-ErbB2 tumorigenesis. Cancer Cell. 2010;17(1):65-76.

18. Kato J, Matsushime H, Hiebert SW, Ewen ME, Sherr CJ. Direct binding of cyclin $\mathrm{D}$ to the retinoblastoma gene product $(\mathrm{pRb})$ and $\mathrm{pRb}$ phosphorylation by the cyclin D-dependent kinase CDK4. Genes Dev. 1993; 7(3):331-342.

19. Alao JP. The regulation of cyclin D1 degradation: roles in cancer development and the potential for therapeutic invention. Mol Cancer. 2007;6:24.

20. Molenaar JJ, Ebus ME, Koster J, et al. Cyclin D1 and CDK4 activity contribute to the undifferentiated phenotype in neuroblastoma. Cancer Res. 2008;68(8):2599-2609.

21. Noel EE, Yeste-Velasco M, Mao X, et al. The association of CCND1 overexpression and cisplatin resistance in testicular germ cell tumors and other cancers. Am J Pathol. 2010;176(6):2607-2615.

22. Gan L, Liu P, Lu H, et al. Cyclin D1 promotes anchorage-independent cell survival by inhibiting FOXO-mediated anoikis. Cell Death Differ. 2009;16(10):1408-1417.

23. Huang $\mathrm{H}, \mathrm{Hu} \mathrm{YD}, \mathrm{Li} \mathrm{N}, \mathrm{Zhu}$ Y. Inhibition of tumor growth and metastasis by non-small cell lung cancer cells transfected with cyclin D1-targeted siRNA. Oligonucleotides. 2009;19(2):151-162.

24. Dalerba P, Cho RW, Clarke MF. Cancer stem cells: models and concepts. Annu Rev Med. 2007;58:267-284.

25. Fong MY, Kakar SS. The role of cancer stem cells and the side population in epithelial ovarian cancer. Histol Histopathol. 2010;25(1): $113-120$.

26. Ahmed N, Abubaker K, Findlay J, Quinn M. Epithelial mesenchymal transition and cancer stem cell-like phenotypes facilitate chemoresistance in recurrent ovarian cancer. Curr Cancer Drug Targets. 2010;10(3):268-278.

27. Morel AP, Lièvre M, Thomas C, Hinkal G, Ansieau S, Puisieux A. Generation of breast cancer stem cells through epithelial-mesenchymal transition. PLoS One. 2008;3(8):e2888. 
28. Mueller T, Luetzkendorf J, Nerger K, Schmoll HJ, Mueller LP. Analysis of OCT4 expression in an extended panel of human tumor cell lines from multiple entities and in human mesenchymal stem cells. Cell Mol Life Sci. 2009;66(3):495-503.

29. Hombach-Klonisch S, Paranjothy T, Wiechec E, et al. Cancer stem cells as targets for cancer therapy: selected cancers as examples. Arch Immunol Ther Exp (Warsz). 2008;56(3):165-180.

30. Chen YC, Hsu HS, Chen YW, et al. Oct-4 expression maintained cancer stem-like properties in lung cancer-derived CD133-positive cells. Plos One. 2008;3(7):e2637.

31. Liu J, Cao L, Chen JC, et al. Bmi1 regulates mitochondrial function and the DNA damage response pathway. Nature. 2009;459(7245):387-392.

32. Sangiorgi E, Capecchi MR. Bmil is expressed in vivo in intestinal stem cells. Nat Genet. 2008;40(7):915-920.

33. Cui HJ, Hu B, Li T, et al. Bmi-1 is essential for the tumorigenicity of neuroblastoma cells. Am J Pathol. 2007;170(4):1370-1378.

34. Thiery JP, Acloque H, Huang RY, Nieto MA. Epithelial-mesenchymal transitions in development and disease. Cell. 2009;139(5):871-890.

35. Acloque H, Adams MS, Fishwick K, Bronner-Fraser M, Nieto MA. Epithelial-mesenchymal transitions: the importance of changing cell state in development and disease. J Clin Invest. 2009;119(6):1438-1449.

36. Hugo H, Ackland ML, Blick T, et al. Epithelial - mesenchymal and mesenchymal - epithelial transitions in carcinoma progression. J Cell Physiol. 2007;213(2):374-383.
37. Chaffer CL, Brennan JP, Slavin JL, Blick T, Thompson EW, Williams ED. Mesenchymal-to-epithelial transition facilitates bladder cancer metastasis: role of fibroblast growth factor receptor-2. Cancer Res. 2006;66(23):11271-11278.

38. Lee TK, Poon RT, Yuen AP, et al. Twist overexpression correlates with hepatocellular carcinoma metastasis through induction of epithelialmesenchymal transition. Clin Cancer Res. 2006;12(18):5369-5376.

39. Alonso SR, Tracey L, Ortiz P, et al. A high-throughput study in melanoma identifies epithelial-mesenchymal transition as a major determinant of metastasis. Cancer Res. 2007;67(7):3450-3460.

40. Shield K, Ackland ML, Ahmed N, Rice GE. Multicellular spheroids in ovarian cancer metastases: biology and pathology. Gynecol Oncol. 2009;113(1):143-148.

41. Patel IS, Madan P, Getsios S, Bertrand MA, MacCalman CD. Cadherin switching in ovarian cancer progression. Int J Cancer. 2003;106(2): $172-177$.

42. Sundfeldt K. Cell-cell adhesion in the normal ovary and ovarian tumors of epithelial origin: an exception to the rule. Mol Cell Endocrinol. 2003;202(1-2):89-96.

43. Frisch SM, Ruoslahti E. Integrins and anoikis. Curr Opin Cell Biol. 1997;9(5):701-706.
OncoTargets and Therapy

\section{Publish your work in this journal}

OncoTargets and Therapy is an international, peer-reviewed, open access journal focusing on the pathological basis of all cancers, potential targets for therapy and treatment protocols employed to improve the management of cancer patients. The journal also focuses on the impact of management programs and new therapeutic agents and protocols on

\section{Dovepress}

patient perspectives such as quality of life, adherence and satisfaction. The manuscript management system is completely online and includes a very quick and fair peer-review system, which is all easy to use. Visit http://www.dovepress.com/testimonials.php to read real quotes from published authors. 